\title{
Влияние легирования редкоземельными элементами (Eu, Tb, Dy) на электропроводность слоистых монокристаллов $\mathrm{Bi}_{2} \mathrm{Te}_{3}$
}

\author{
(C) Н.А. Абдуллаев ${ }^{1, \uparrow}$, К.М. Джафрарли ${ }^{1}$, Х.В. Алигулиева ${ }^{1}$, Л.Н. Алиева ${ }^{1}$, \\ C.Ш. Кахраманов ${ }^{2}$, C.A. Немов ${ }^{3}$ \\ ${ }^{1}$ Институт фризики Национальной академии Азербайджана, \\ AZ-1143 Баку, Азербайджан \\ ${ }^{2}$ Азербайджанский технический университет, \\ AZ-1173 Баку, Азербайджан \\ ${ }^{3}$ Санкт-Петербургский государственный политехнический университет, \\ 195251 Санкт-Петербург, Россия \\ E-mail: abnadir@physics.ab.az
}

(Получена 12 декабря 2016 г. Принята к печати 26 декабря 2016 г.)

\begin{abstract}
Исследованы температурные зависимости в интервале температур $T=5-300 \mathrm{~K}$ удельного сопротивления в плоскости слоев и в направлении, перпендикулярном слоям, а также эффекты Холла и поперечного магнитосопротивления в нелегированных и легированных слоистых монокристаллах $\mathrm{Bi}_{2} \mathrm{Te}_{3}$ (магнитные поля $<80$ КЭ, $T=5 \mathrm{~K}$ ). Показано, что при легировании $\mathrm{Bi}_{2} \mathrm{Te}_{3}$ атомами редкоземельных элементов Еu, $\mathrm{Tb}$, Dy наблюдается увеличение удельного сопротивления как в плоскости слоев, так и в направлении, перпендикулярном слоям $\mathrm{Bi}_{2} \mathrm{Te}_{3}$. Увеличение удельного сопротивления обусловлено главным образом уменьшением подвижности носителей заряда вследствие повышения роли в рассеянии процессов рассеяния носителей на дефектах. Оценены величины концентраций и подвижностей носителей заряда, а также значения холл-фактора, обусловленного анизотропией эффективных масс и ориентацией эллипсоидов относительно кристаллографических осей.
\end{abstract}

DOI: $10.21883 /$ FTP.2017.07.44658.8483

\section{1. Введение}

Как известно, соединения группы $\mathrm{A}_{2}^{\mathrm{V}} \mathrm{B}_{3}^{\mathrm{VI}}$ и твердые растворы на их основе являются широко распространенными материалами для создания миниатюрных, экологически чистых, бесшумных термоэлектрических преобразователей (холодильников, термогенераторов и др.). Одним из способов повышения термоэлектрической эффективности $Z\left(Z=S^{2} \sigma / \chi\right.$, где $S-$ коэффициент Зеебека, $\sigma$ - проводимость, $\chi-$ коэффициент теплопроводности) является легирование этих материалов различными элементами, при котором возможно добиться некоторого увеличения коэффициента термоэдс и проводимости с одновременным падением величины теплопроводности вследствие рассеяния фононов на дефектах. В настоящей работе была исследована электропроводность слоистых монокристаллов $\mathrm{Bi}_{2} \mathrm{Te}_{3}$, легированных примесями редкоземельных элементов Еu, $\mathrm{Tb}$, Dу. Использование в качестве примеси атомов редкоземельных элементов (РЗЭ) обусловлено несколькими причинами. Согласно $[1,2]$, поведение примесей Р3Э в полупроводниках характеризуется некоторыми отличительными особенностями, например, сочетанием малой растворимости и способности РЗЭ производить „очистку“ материала, при которой возможно значительное снижение концентрации фоновых примесей и дефектов и, как следствие, увеличение подвижности носителей заряда. Замещение атомов висмута атомами Р3Э должно оказывать акцепторное действие, так как в атоме висмута валентных электронов больше, что приводит к увеличению концентрации дырок в $\mathrm{Bi}_{2} \mathrm{Te}_{3}$.
Кроме того, ионные радиусы атомов РЗЭ близки к радиусу иона висмута.

\section{2. Эксперимент}

Монокристаллы $\mathrm{Bi}_{2} \mathrm{Te}_{3}$ выращивались методом Бриджмена и вертикальной направленной кристаллизацией из компонент со стехиометрическим соотношением и примесями $\mathrm{Eu}, \mathrm{Tb}, \mathrm{Dy}$. Концентрация вводимых примесей составляла $1-2$ ат\%. Зоннонаправленная кристаллизация проводилась при скорости перемещения зоны $3 \mathrm{~cm} /$ ч. Рентгенструктурные исследования образцов проводились на рентгеновском дифрактометре Bruker D8 Advance. Однородность образцов и характерные частоты активных в рамановском рассеянии фононов контролировались на рамановском конфокальном микроспектрометре Nanofinder 30 (Tokyo Instr., Japan).

Как известно, $\mathrm{Bi}_{2} \mathrm{Te}_{3}$ при синтезе из расплава стехиометрического состава характеризуется наличием значительного количества собственных точечных дефектов структуры, обусловленных переходом атомов Ві в позиции Те в решетке $\left(\mathrm{Bi}_{\mathrm{Te}}\right)$. Поскольку такие антиструктурные дефекты являются акцепторами, кристаллы теллурида висмута изначально обладают высокой концентрацией дырок, $p \sim\left(10^{18}-10^{19}\right) \mathrm{cm}^{-3}$. О высоком качестве нелегированных слоистых монокристаллов $\mathrm{Bi}_{2} \mathrm{Te}_{3}$ косвенно свидетельствовало достаточно низкое значение сопротивления образцов, что обусловлено высокой подвижностью носителей заряда, и четкая картина квантовых осцилляций большой амплитуды поперечного магнитосопротивления. 
Исследования проводились в интервале температур $T=5-300 \mathrm{~K}$ и магнитных полях вплоть до 8 Тл. Измерения проводились по селективной методике на переменном токе частотой 20.5 Гц, величина тока не превышала 1 мА. Образцы для исследований получались из монокристаллических слитков простым скалыванием вдоль поверхности, содержащей плоскость слоя, и приготавливались в форме прямоугольных пластин с толщинами 0.2-0.8 мм. При гальваномагнитных исследованиях образец помещался в центр сверхпроводящего соленоида, магнитное поле было направлено перпендикулярно плоскости слоя.

\section{3. Результаты исследований и их обсуждение}

\section{1. Эффект Холла}

Измерения эффекта Холла в легированных монокристаллах $\mathrm{Bi}_{2} \mathrm{Te}_{3}$ позволяют не только оценить концентрацию носителей заряда, но и получить информацию относительно возможного изменения структуры валентной зоны (наличия примесных уровней) при легировании различными примесями. В слабом магнитном поле на эффект Холла оказывают влияние механизмы рассеяния и степень вырождения газа носителей заряда. С учетом этого коэффициент Холла при слабом магнитном поле, направленном перпендикулярно плоскости слоя, есть [3]

$$
\left|R_{\perp}\right|=\frac{A\left(r, \zeta^{*}\right)}{n e} B_{\perp}
$$

где $A\left(r, \zeta^{*}\right)$ зависит от механизма рассеяния и степени вырождения газа свободных носителей, $B_{\perp}-$ фактор анизотропии, равный для монокристаллов $\mathrm{Bi}_{2} \mathrm{Te}_{3}$ $0.3-0.4, n-$ концентрация носителей заряда, $e-$ заряд электрона. В сильном магнитном поле $B$ (при $\mu B \gg 1$, $\mu-$ магнитная проницаемость) предельное значение коэффициента Холла равно

$$
|R(\infty)|=\frac{1}{n e}
$$

как и в случае простой изотропной модели энергетического спектра. Измерения эффекта Холла нами были проведены в достаточно сильных магнитных полях, вплоть до 1 Тл, при которых справедливо соотношение (2).

Как отмечалось выше, изначально монокристаллы $\mathrm{Bi}_{2} \mathrm{Te}_{3}$ имеют $p$-тип проводимости. В нелегированных образцах концентрация дырок достигала $\sim 6.6 \cdot 10^{18} \mathrm{~cm}^{-3}$. Из данных исследования эффекта Холла следует, что легирование монокристаллов $\mathrm{Bi}_{2} \mathrm{Te}_{3}$ элементом $\mathrm{Eu}$ приводит к возрастанию концентрации дырок до $2 \cdot 10^{19} \mathrm{~cm}^{-3}$, и это означает, что европий оказывает в $\mathrm{Bi}_{2} \mathrm{Te}_{3}$ в основном акцепторное действие. Атомы европия образуют при легировании $\mathrm{Bi}_{2} \mathrm{Te}_{3}$ твердые растворы замещения, занимая позиции атомов висмута. Поскольку такое замещение сопровождается дефицитом валентных электронов, европий оказывает в $\mathrm{Bi}_{2} \mathrm{Te}_{3}$ акцепторное действие.

Почти то же самое происходит при легировании монокристаллов $\mathrm{Bi}_{2} \mathrm{Te}_{3}$ элементом Тb. В легированных образцах концентрация дырок возрастает до $\sim 7 \cdot 10^{19} \mathrm{~cm}^{-3}$, что свидетельствует об акцепторном действии тербия в $\mathrm{Bi}_{2} \mathrm{Te}_{3}$. Вероятно, в этом случае атомы тербия также образуют при легировании в $\mathrm{Bi}_{2} \mathrm{Te}_{3}$ твердые растворы замещения, занимая позиции атомов висмута.

Однако, в отличие от элементов $\mathrm{Eu} \mathrm{и} \mathrm{Tb}$, при легировании монокристаллов $\mathrm{Bi}_{2} \mathrm{Te}_{3}$ элементом Dу характер проводимости меняется на $n$-тип, что свидетельствует о донорном действии диспрозия в $\mathrm{Bi}_{2} \mathrm{Te}_{3}$. Из данных исследования эффекта Холла следует, что легирование монокристаллов $\mathrm{Bi}_{2} \mathrm{Te}_{3}$ элементом Dу приводит к концентрации электронов $3 \cdot 10^{18} \mathrm{~cm}^{-3}$. Вероятно, в этом случае атомы диспрозия образуют при легировании в $\mathrm{Bi}_{2} \mathrm{Te}_{3}$ твердые растворы внедрения, отдавая валентные электроны в зону проводимости.

\section{2. Электропроводность}

Абсолютные значения удельного сопротивления в плоскости слоев $\left(\rho_{\mathrm{par}}\right)$ и перпендикулярно слоям $\left(\rho_{\mathrm{per}}\right)$ определялись усовершенствованным четырехзондовым комбинационным методом Шнабеля [3], разработанным специально для определения анизотропии проводимости слоистых кристаллов. Точечные контакты при таких измерениях наносились попарно на противоположные стороны пластинки в прямоугольной геометрии.

На рис. 1 и 2 приведены рассчитанные температурные зависимости абсолютных значений удельных сопротивлений в характерных кристаллографических направлениях (в плоскости слоев $\rho_{\text {par }}$ и перпендикулярно слоям $\rho_{\text {per }}$ ) нелегированного $\mathrm{Bi}_{2} \mathrm{Te}_{3}$ (кривые 1 ) и легированного европием (кривые 2), тербием (кривые 3) и диспрозием (кривые 4). Как видно из рис. 1 и 2, общими для всех образцов являются:

1) характерный „металлический“ ход температурной зависимости сопротивления - с понижением температуры сопротивление падает, а при низких температурах выходит на плато;

2) при низких температурах остаточное сопротивление наименьшее у нелегированного $\mathrm{Bi}_{2} \mathrm{Te}_{3}$ (кривые 1), что обусловлено увеличением рассеяния носителей заряда на дефектах в легированных образцах.

Поскольку концентрация носителей заряда в образцах с температурой практически не изменялась, все изменение сопротивления обусловлено изменением подвижности носителей заряда. При температурах > $100 \mathrm{~K}$ преобладает рассеяние носителей заряда на колебаниях кристаллической решетки, при $30 \mathrm{~K}$ в области почти постоянной электропроводности примесное рассеяние преобладает над решеточным. Подвижность носителей заряда в этом случае должна зависеть от температуры только через энергию носителей, а последняя при силь- 


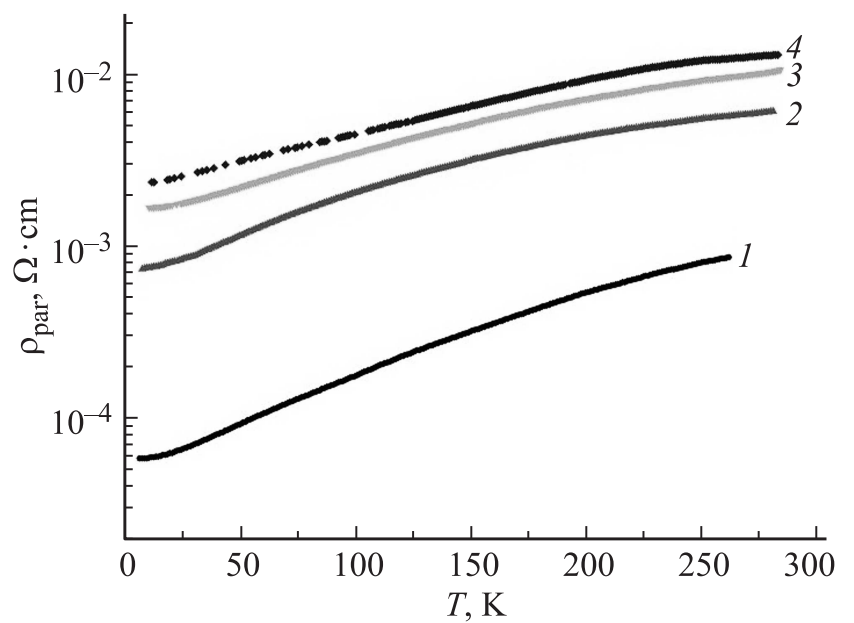

Рис. 1. Температурные зависимости удельного сопротивления в плоскости слоев $\left(\rho_{\text {par }}\right)$ легированных и нелегированных слоистых монокристаллов в области температур $5<T<300 \mathrm{~K}$ : 1 - нелегированный $\mathrm{Bi}_{2} \mathrm{Te}_{3}, 2-\mathrm{Bi}_{2} \mathrm{Te}_{3}\langle\mathrm{Eu}\rangle, 3-\mathrm{Bi}_{2} \mathrm{Te}_{3}\langle\mathrm{~Tb}\rangle$, $4-\mathrm{Bi}_{2} \mathrm{Te}_{3}\langle\mathrm{Dy}\rangle$.

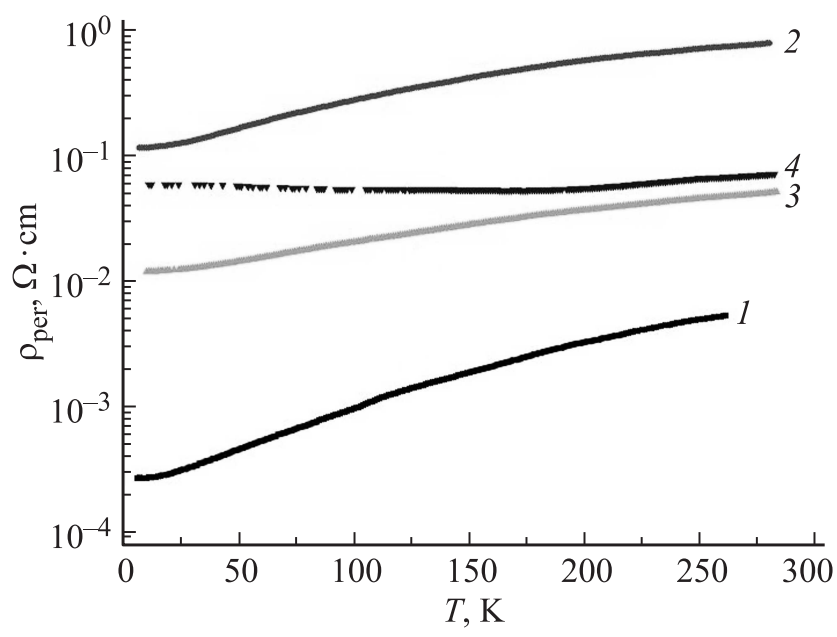

Рис. 2. Температурные зависимости удельного сопротивления в направлении, перпендикулярном слоям $\left(\rho_{\text {per }}\right)$, легированных и нелегированных слоистых монокристаллов в области температур $5<T<300 \mathrm{~K}: 1-$ нелегированный $\mathrm{Bi}_{2} \mathrm{Te}_{3}, 2-$ $\mathrm{Bi}_{2} \mathrm{Te}_{3}\langle\mathrm{Eu}\rangle, 3-\mathrm{Bi}_{2} \mathrm{Te}_{3}\langle\mathrm{~Tb}\rangle, 4-\mathrm{Bi}_{2} \mathrm{Te}_{3}\langle\mathrm{Dy}\rangle$.

ном вырождении практически не меняется с температурой и потому сопротивление при низких температурах выходит на плато.

Анализ данных рис. 3 и 4 показывает, что в нелегированных образцах $\mathrm{Bi}_{2} \mathrm{Te}_{3}$ при температурах $T \approx 150-300 \mathrm{~K}$ подвижность в плоскости слоев меняется с температурой как $\mu_{\|}(T) \propto T^{-1.7}$, а в перпендикулярном направлении как $\mu_{\perp}(T) \propto T^{-1.78}$. Как известно, в случае чисто акустического рассеяния носителей заряда в рамках классической статистики подвижность $\propto T^{-1.5}$. Однако в слоистых кристаллах, в отличие от изотропных кристаллов, из-за наличия слабой связи растяжение в одном направлении не сопровождается эквивалентным сжатием в другом. Появляются уже существенные градиенты атомных потенциалов и потенциал рассеяния не равен нулю. В этом случае рассеяние носителей заряда на оптических фононах значительно, что приводит к более резкой температурной зависимости подвижности.

Иначе обстоит дело в образцах $\mathrm{Bi}_{2} \mathrm{Te}_{3}$, легированных европием, тербием и диспрозием. В таких образцах температурная зависимость подвижностей, как в плоскости слоев (рис. 3), так и перпендикулярно слоям (рис. 4), значительно ослаблена. $\mathrm{B} \mathrm{Bi}_{2} \mathrm{Te}_{3}$, легированном европием, подвижность носителей заряда в плоскости слоев меняется с температурой как $\mu_{\|}(T) \propto T^{-1.04}$ $(T=80-300 \mathrm{~K}), \quad$ перпендикулярно слоям $\mu_{\perp}(T) \propto T^{-0.99}(T=70-300 \mathrm{~K}) ;$ в $\mathrm{Bi}_{2} \mathrm{Te}_{3}$, легированном тербием, подвижности меняются как $\mu_{\|}(T) \propto T^{-1.07}$

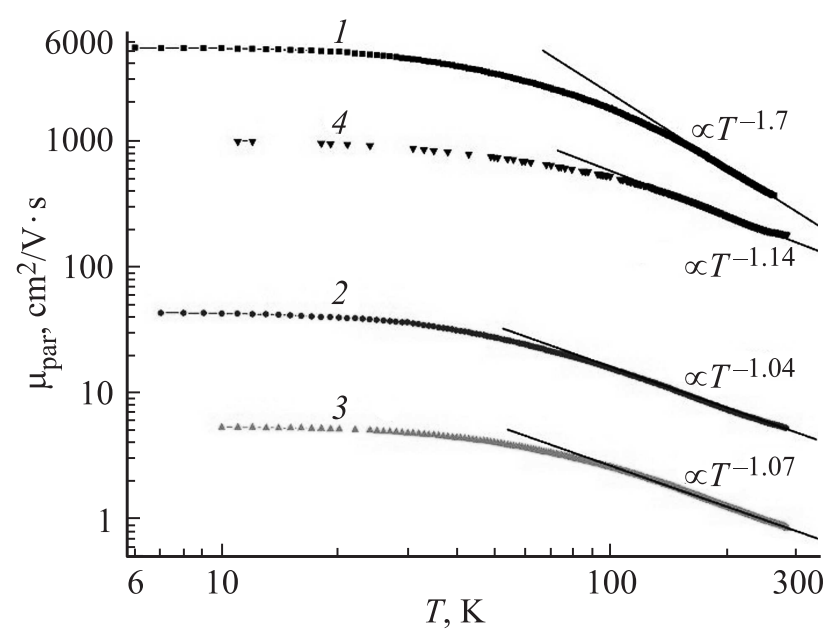

Рис. 3. Температурные зависимости подвижности носителей заряда в плоскости слоев $\left(\mu_{\mathrm{par}}\right)$ легированных и нелегированных слоистых монокристаллов в области температур $5<T<300 \mathrm{~K}: 1-$ нелегированный $\mathrm{Bi}_{2} \mathrm{Te}_{3}, 2-\mathrm{Bi}_{2} \mathrm{Te}_{3}\langle\mathrm{Eu}\rangle$, $3-\mathrm{Bi}_{2} \mathrm{Te}_{3}\langle\mathrm{~Tb}\rangle, 4-\mathrm{Bi}_{2} \mathrm{Te}_{3}\langle\mathrm{Dy}\rangle$.

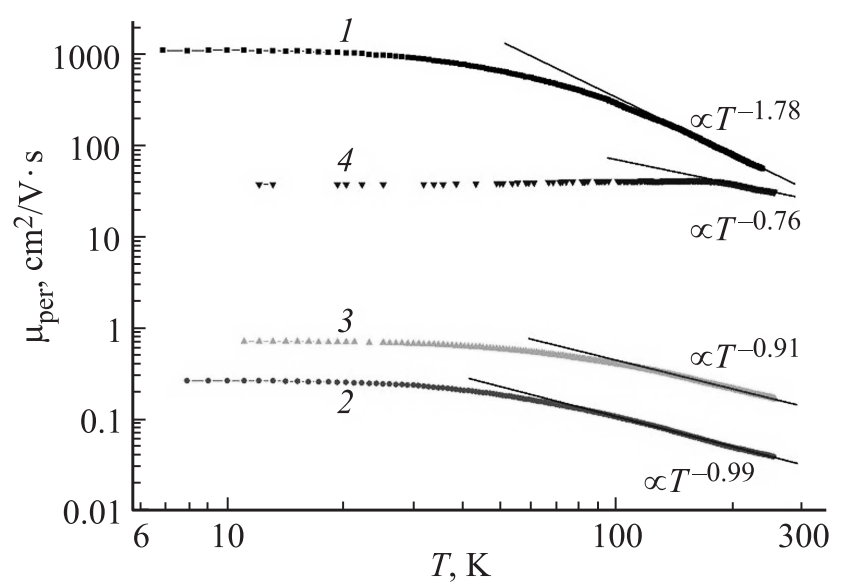

Рис. 4. Температурные зависимости подвижности носителей заряда в направлении, перпендикулярном слоям $\left(\mu_{\mathrm{per}}\right)$, легированных и нелегированных слоистых монокристаллов в области температур $5<T<300 \mathrm{~K}: 1-$ нелегированный $\mathrm{Bi}_{2} \mathrm{Te}_{3}, 2-$ $\mathrm{Bi}_{2} \mathrm{Te}_{3}\langle\mathrm{Eu}\rangle, 3-\mathrm{Bi}_{2} \mathrm{Te}_{3}\langle\mathrm{~Tb}\rangle, 4-\mathrm{Bi}_{2} \mathrm{Te}_{3}\langle\mathrm{Dy}\rangle$. 


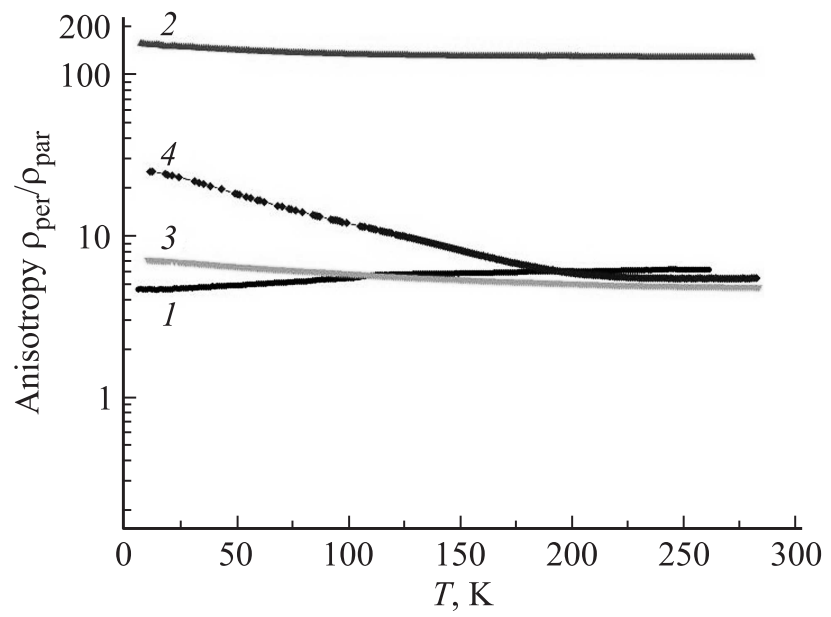

Рис. 5. Температурные зависимости анизотропии удельного сопротивления легированных и нелегированных монокристаллов $\mathrm{Bi}_{2} \mathrm{Te}_{3}$ в области температур $5<T<300 \mathrm{~K}$ : 1 - нелегированный $\mathrm{Bi}_{2} \mathrm{Te}_{3}, 2-\mathrm{Bi}_{2} \mathrm{Te}_{3}\langle\mathrm{Eu}\rangle, 3-\mathrm{Bi}_{2} \mathrm{Te}_{3}\langle\mathrm{~Tb}\rangle$, $4-\mathrm{Bi}_{2} \mathrm{Te}_{3}\langle\mathrm{Dy}\rangle$.

$(T=90-300 \mathrm{~K}), \quad \mu_{\perp}(T) \propto T^{-0.91} \quad(T=100-300 \mathrm{~K}), \quad$ в $\mathrm{Bi}_{2} \mathrm{Te}_{3}$, легированном диспрозием, подвижности меняются как $\mu_{\|}(T) \propto T^{-1.14}(T=110-300 \mathrm{~K}), \mu_{\perp}(T) \propto T^{-0.76}$ $(T=180-300 \mathrm{~K})$. Ослабление температурной зависимости подвижности, по-видимому, обусловлено увеличением роли примесного рассеяния в легированных образцах.

Из рис. 4 видно, что в $\mathrm{Bi}_{2} \mathrm{Te}_{3}$, легированном европием (кривая 2), подвижность носителей заряда в направлении, перпендикулярном слоям, наименьшая. Повидимому, при легировании слоистого $\mathrm{Bi}_{2} \mathrm{Te}_{3}$ часть атомов примеси располагается в промежутках между слоями из-за плотной упаковки атомов внутри слоевквинтетов. При этом такие дефекты, относительно слабо влияя на движение носителей заряда в плоскости слоев, существенно затрудняют их движение поперек слоям. Благодаря таким дефектам (дефектам стыковки слоев) наблюдается значительная анизотропия проводимости, не соответствующая анизотропии эффективных масс, во многих слоистых полупроводниках - например, в InSe [4]. На рис. 5 показано температурное изменение анизотропии удельных сопротивлений $\rho_{\text {per }} / \rho_{\text {par. }}$. Как видно из рисунка, в нелегированном $p-\mathrm{Bi}_{2} \mathrm{Te}_{3}$ существует анизотропия электропроводности $\rho_{\mathrm{per}} / \rho_{\mathrm{par}} \sim 5$, почти не меняющаяся с температурой, что согласуется с известными литературными данными $[5,6]$, а в $\mathrm{Bi}_{2} \mathrm{Te}_{3}$, легированном европием (кривая 2), анизотропия электропроводности наибольшая.

\section{3. Магнитосопротивление в области классических магнитных полей}

При исследованиях магнитосопротивления в слоистом $\mathrm{Bi}_{2} \mathrm{Te}_{3}$ ток протекал в плоскости слоев, а магнитное поле было направлено перпендикулярно плоскости слоев. Полевая зависимость магнитосопротивления $\rho_{x x}$ в нелегированном $\mathrm{Bi}_{2} \mathrm{Te}_{3}$ (рис. 6) характерна для идеальных полупроводников [7]: в слабых магнитных полях наблюдается квадратичный рост $\rho_{x x}$, а в сильных полях наблюдаются осцилляции магнитосопротивления. В [8], в рамках общепринятой шестиэллипсоидной модели зонной структуры $\mathrm{Bi}_{2} \mathrm{Te}_{3}$ Дреббла-Вольфа [9] получены формулы, описывающие зависимость поперечного удельного сопротивления $\rho_{x x}$ от магнитного поля $B$. С учетом того, что магнитное поле направлено перпендикулярно слоям, т.е. направлено вдоль оси кристаллографической $\mathbf{C}_{3}$, при сильном вырождении газа носителей заряда имеем согласно [8]

$$
\frac{\rho_{x x}(B)}{\rho_{0}}=\frac{1+\left(R_{0} \sigma_{0} B\right)^{2} / f_{\|}}{1+\left(R_{0} \sigma_{0} B\right)^{2}},
$$

где $\rho_{0}$ и $\sigma_{0}-$ значения удельного сопротивления и проводимости при $B=0, R_{0}=\rho_{123}$ при $B \rightarrow 0$, а $f_{\|}-$ холл-фактор, обусловленный анизотропией эффективных масс и ориентацией эллипсоидов относительно кристаллографических осей. Для удобства (1) можно переписать как функцию $B^{2} /\left(\Delta \rho / \rho_{0}\right)$ от $B^{2}$ :

$$
\frac{B^{2}}{\left(\Delta \rho / \rho_{0}\right)}=\frac{1}{\left(1 / f_{\|}-1\right)\left(R_{0} \sigma_{0}\right)^{2}}+\frac{1}{\left(1 / f_{\|}-1\right)} B^{2},
$$

где $\Delta \rho=\rho_{x x}-\rho_{0}$.

На рис. 7 приведены магнитополевые зависимости поперечного сопротивления в координатах $B^{2} /\left(\Delta \rho / \rho_{0}\right)$ от $B^{2}$ для нелегированных монокристаллов $\mathrm{Bi}_{2} \mathrm{Te}_{3}$ (кривая 1), легированных $\mathrm{Bi}_{2} \mathrm{Te}_{3}\langle\mathrm{Eu}\rangle$ (кривая 2), $\mathrm{Bi}_{2} \mathrm{Te}_{3}\langle\mathrm{~Tb}\rangle$ (кривая 3) и $\mathrm{Bi}_{2} \mathrm{Te}_{3}\langle\mathrm{Dy}\rangle$ (кривая 4). Из (2) возможно определить по тангенсу угла наклона прямой сначала холл-фактор $f_{\|}$, а затем из отрезка, отсекаемого прямой на оси ординат, и холловскую подвижность $\mu_{\mathrm{H}}=R_{0} \sigma_{0}$. Определенные таким образом значения холл-фактора $f_{\|}$ и холловской подвижности $\mu_{\mathrm{H}}$ примерно равны 0.37 и $2700 \mathrm{~cm}^{2} / \mathrm{B} \cdot \mathrm{c} \mathrm{в} \mathrm{Bi}_{2} \mathrm{Te}_{3}, 0.28$ и $1700 \mathrm{~cm}^{2} / \mathrm{B} \cdot \mathrm{c} \mathrm{в} \mathrm{Bi}_{2} \mathrm{Te}_{3}\langle\mathrm{Eu}\rangle$,

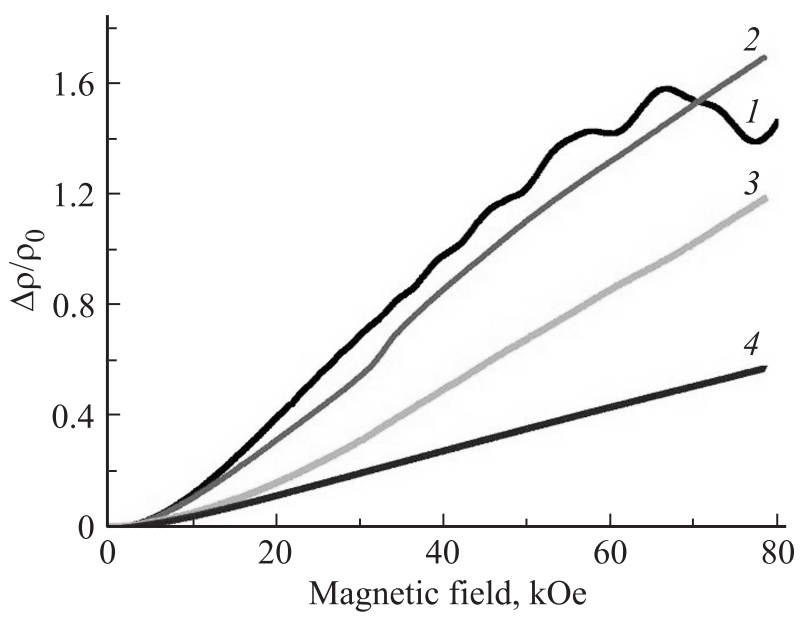

Рис. 6. Магнитополевая зависимость сопротивления: 1 - нелегированный $\mathrm{Bi}_{2} \mathrm{Te}_{3}, 2-\mathrm{Bi}_{2} \mathrm{Te}_{3}\langle\mathrm{Eu}\rangle, 3-\mathrm{Bi}_{2} \mathrm{Te}_{3}\langle\mathrm{~Tb}\rangle$, $4-\mathrm{Bi}_{2} \mathrm{Te}_{3}\langle\mathrm{Dy}\rangle$. 


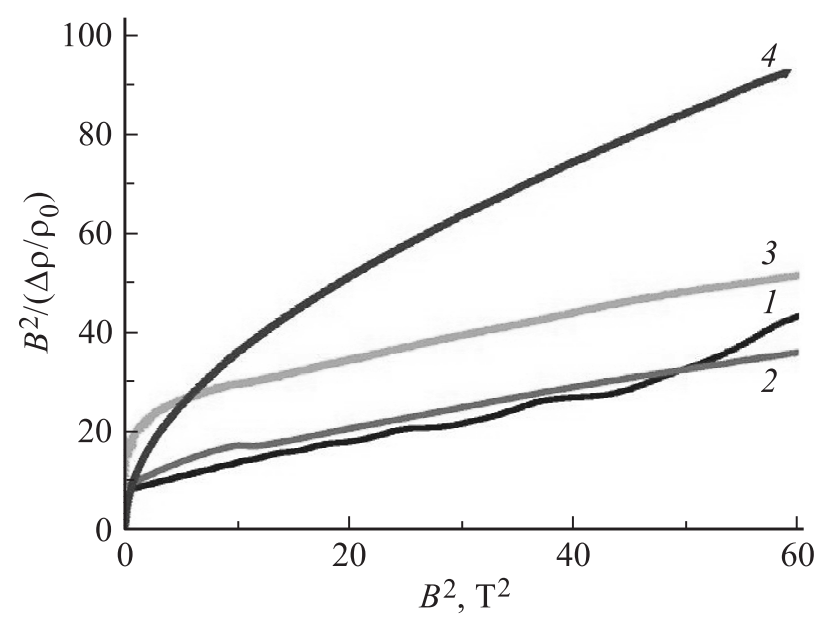

Рис. 7. Магнитополевая зависимость сопротивления в координатах $B^{2} /\left(\Delta \rho / \rho_{0}\right)-B^{2}: 1-$ нелегированный $\mathrm{Bi}_{2} \mathrm{Te}_{3}$, $2-\mathrm{Bi}_{2} \mathrm{Te}_{3}\langle\mathrm{Eu}\rangle, 3-\mathrm{Bi}_{2} \mathrm{Te}_{3}\langle\mathrm{~Tb}\rangle, 4-\mathrm{Bi}_{2} \mathrm{Te}_{3}\langle\mathrm{Dy}\rangle$.

0.33 и $1300 \mathrm{~cm}^{2} / \mathrm{B} \cdot \mathrm{c}$ в $\mathrm{Bi}_{2} \mathrm{Te}_{3}\langle\mathrm{~Tb}\rangle, 0.5$ и $1700 \mathrm{~cm}^{2} / \mathrm{B} \cdot \mathrm{c}$ в $\mathrm{Bi}_{2} \mathrm{Te}_{3}\langle\mathrm{Dy}\rangle$. Как видно из приведенных результатов, величины холл-факторов $f_{\|}$лежат в пределах $0.3-0.5$, что находится в согласии с литературными данными [8]. Это свидетельствует об отсутствии заметного влияния примесей на зонные параметры дырок в $\mathrm{Bi}_{2} \mathrm{Te}_{3}$. Надо отметить, что оцененные из (2) холловские подвижности носителей заряда $\mu_{\mathrm{H}}$ оказываются выше, чем определенные из экспериментов по исследованиям электропроводности и эффекта Холла. Вероятно, это связано с тем, что соотношение (1) получено теоретически из шестиэллипсоидной модели зонной структуры $\mathrm{Bi}_{2} \mathrm{Te}_{3}$ без учета реальной структуры и дефектов.

\section{4. Заключение}

Из приведенных выше данных следует, что легирование монокристаллов $\mathrm{Bi}_{2} \mathrm{Te}_{3}$ атомами редкоземельных элементов $\mathrm{Eu}, \mathrm{Tb}$, Dy приводит к увеличению сопротивления как в плоскости слоев, так и в направлении, перпендикулярном слоям, главным образом за счет уменьшения подвижности носителей заряда вследствие примесного рассеяния. Об этом свидетельстует также ослабление температурной зависимости подвижности и увеличение остаточного сопротивления при низких температурах. Легирование $\mathrm{Bi}_{2} \mathrm{Te}_{3}$ атомами $\mathrm{Eu}, \mathrm{Tb}$ происходит в основном по принципу замещения атомов висмута атомами $\mathrm{Eu}, \mathrm{Tb}$, что приводит к увеличению концентрации дырок: в $\mathrm{Bi}_{2} \mathrm{Te}_{3}\langle\mathrm{Eu}\rangle$ до 2. $10^{19} \mathrm{~cm}^{-3}$, в $\mathrm{Bi}_{2} \mathrm{Te}_{3}\langle\mathrm{~Tb}\rangle$ до $7 \cdot 10^{19} \mathrm{~cm}^{-3}$. Однако в $\mathrm{Bi}_{2} \mathrm{Te}_{3}\langle\mathrm{Dy}\rangle$ наблюдается изменения типа проводимости с дырочной на электронную, при этом концентрация электронов $\sim 3 \cdot 10^{18} \mathrm{~cm}^{-3}$. Повидимому, в этом случае атомы диспрозия образуют при легировании $\mathrm{Bi}_{2} \mathrm{Te}_{3}$ твердые растворы внедрения, отдавая валентные электроны в зону проводимости.

К сожалению, при исследованиях легированных материалов трудно привести достоверную зависимость полученных данных от концентрации вводимых примесей вследствие неконтролируемых потерь в технологическом процессе, адсорбции примеси стенками ампулы, неравномерного распределения примеси по длине слитка при направленной кристаллизации, выделения на дислокациях и границах блоков и др.

Работа выполнена благодаря финансовой поддержке Фонда развития науки при президенте Азербайджанской республики (грант № EIF/GAM-3-2014-6(21)-24/01/1 и гранта № EIF/MQM/Elm-Təhsil-1-2016-1(26)).

\section{Список литературы}

[1] В.Ф. Мастеров, Л.Ф. Захаренков. ФТП, 24 (4), 610 (1990).

[2] Б.М. Гольцман, В.А. Кудинов, И.А. Смирнов. Полупроводниковые термоэлектрические материалы на основе $\mathrm{Bi}_{2} \mathrm{Te}_{3}$ (М., Наука, 1972).

[3] P. Schnabel. Zeits. Angew. Phys., 22 (2), 136 (1967).

[4] Г.Л. Беленький, Н.А. Абдуллаев, В.Н. Зверев, В.Я. Штейншрайбер. Письма ЖЭТФ, 47 (10), 498 (1988).

[5] Б.А. Ефимова, И.Я. Коренблит, В.И. Новиков, А.Г. Остроумов. ФТТ, 3 (9), 2746 (1961).

[6] R.T. Delves, A.E. Bowley, D.W. Haselden, H.J. Goldsmid. Proc. Phys. Soc., 78, 838 (1961).

[7] Б.М. Аскеров. Кинетические эфббекты в полупроводниках (Л., Наука, 1970).

[8] Р. Лайхо, С.А. Немов, А.В. Лашкул, Э. Лахдеранта, Т.Е. Свечникова, Д.С. Дворник. ФТП, 41 (5), 565 (2007).

[9] J.R. Drabble, R. Wolfe. Proc. Phys. Soc., 69, 1101 (1956).

Редактор Л.В. Шаронова

\section{Effect of doping with rare earth elements (Eu, Tb, Dy) on the electrical conductivity in $\mathbf{B i}_{2} \mathbf{T e}_{3}$ layered crystals}

N.A. Abdullayev ${ }^{1}$, K.M. Jafarli ${ }^{1}$, Kh.V. Aliguliyeva ${ }^{1}$, L.N. Aliyeva ${ }^{1}$, S.Sh. Kahramanov ${ }^{2}$, S.A. Nemov ${ }^{3}$

${ }^{1}$ Institute of Physics,

Azerbaijan National Academy of Sciences,

AZ-1143 Baku, Azerbaijan

${ }^{2}$ Azerbaijan Technical University,

AZ-1173 Baku, Azerbaijan

${ }^{3}$ Peter the Great St. Petersburg Polytechnic University, 195251 St. Petersburg, Russia

Abstract The temperature dependences (the temperature interval $T=5-300 \mathrm{~K}$ ) of resistivity in the plane of the layers and in the perpendicular direction to the layers, also Hall and the transverse magnetoresistance effects in undoped and doped layered $\mathrm{Bi}_{2} \mathrm{Te}_{3}$ single crystals (magnetic fields $<80 \mathrm{kOe}, T=5 \mathrm{~K}$ ) were investigated. It is shown that upon doping $\mathrm{Bi}_{2} \mathrm{Te}_{3}$ with the rare earth atoms $\mathrm{Eu}, \mathrm{Tb}, \mathrm{Dy}$ an increase of the resistivity in the plane of the layers, as well as in the direction of perpendicular to the layers in $\mathrm{Bi}_{2} \mathrm{Te}_{3}$ was observed. The resistivity increase mainly was caused by decrease of charge carrier mobility as a result of increasing role of the carriers scattering by defects. The charge carriers concentrations and mobilities, values of the Hall factor, conditioned by the anisotropy of effective masses and orientation of ellipsoids with respect to crystallographic axes, were evaluated. 